\title{
Synthesis of Air-Stable CdSe/ZnS Core-Shell Nanoplatelets with Tunable Emission Wavelength
}

Anatolii Polovitsyn, ${ }^{\dagger}{ }^{\dagger}$ Zhiya Dang, ${ }^{\dagger}$ José L. Movilla, $^{\S}$ Beatriz Martín-García, ${ }^{\dagger}$ Ali Hossain Khan, ${ }^{\dagger}$ Guillaume H. V. Bertrand, ${ }^{\dagger, \|}$ Rosaria Brescia, ${ }^{\dagger}$ and Iwan Moreels*, ${ }^{\dagger}$ (1)

${ }^{\dagger}$ Istituto Italiano di Tecnologia, via Morego 30, 16163 Genova, Italy

${ }^{\ddagger}$ Dipartimento di Fisica, Università di Genova, Via Dodecaneso 33, 16146 Genova, Italy

${ }^{\S}$ Departamento de Educación, Universitat Jaume I, Av. Vicent Sos Baynat s/n, 12071 Castellón, Spain

\section{Supporting Information}

\begin{abstract}
In the past few years, several protocols have been reported on the synthesis of $\mathrm{CdSe}$ nanoplatelets with narrow photoluminescence (PL) spectrum, high PL quantum efficiency, and short exciton lifetime. The corresponding core/shell nanoplatelets are however still mostly based on $\mathrm{CdSe} / \mathrm{CdS}$, which possess an extended lifetime and a strong red shift of the band-edge absorption and emission, in accordance with a quasi-type-II band alignment. Here we report on a robust synthesis procedure to grow a $\mathrm{ZnS}$ shell around $\mathrm{CdSe}$ nanoplatelets at moderate temperatures of $100-150{ }^{\circ} \mathrm{C}$, to improve the optical properties of $\mathrm{CdSe}$ nanoplatelets via a type-I core/ shell heterostructure. The shell growth is performed under ambient atmosphere, in either toluene or 1,2-dichlorobenzene. The variation of the shell thickness induces a continuous red shift of the PL peak, eventually reaching $611 \mathrm{~nm}$. The PL quantum efficiency is increased compared to the original $\mathrm{CdSe}$ cores, with values up to $60 \%$ depending on the shell thickness. High-resolution transmission electron microscopy reveals a bending of the nanoplatelets caused by strain due to $12 \%$ lattice mismatch between $\mathrm{CdSe}$ and $\mathrm{ZnS}$. The present procedure can easily be translated to other core/shell nanocrystals, such as $\mathrm{CdSe} / \mathrm{CdS}$ and $\mathrm{CdSe} /$ $\mathrm{CdZnS}$ nanoplatelets.
\end{abstract}

\section{INTRODUCTION}

The first syntheses of colloidal semiconductor nanocrystals (NCs) have been published several decades ago. ${ }^{1-3}$ Due to their small size, they experience strong quantum confinement in all dimensions and are therefore also referred to as quantum dots (QDs). Already early on, their size- and shape-dependent band structure made them excellent candidates to study exciton dynamics in confined systems, ${ }^{4-7}$ and to use them in optoelectronic and photonic applications ${ }^{8,9}$ such as lightemitting diodes, ${ }^{10}$ solar cells and photodetectors, ${ }^{11,12}$ or fluorescent dyes. ${ }^{13}$

Nanometer-sized QDs contain a significant number of surface atoms. The exposure of such a large surface to the environment often leads to oxidation and carrier trapping at the surface, generally leading to a reduced photoluminescence quantum efficiency (PL QE). To prevent this, procedures have been developed to deposit a protective shell of another semiconductor material around the NC core, increasing the PL $\mathrm{QE}$ and reducing the contribution of trap states to the electron-hole recombination. ${ }^{14-17}$ Starting from a CdSe core, typical materials used for the shell growth are $\mathrm{ZnS}^{14,15}$ or CdS. ${ }^{16,17}$ Depending on the choice of core and shell material, the PL QE and carrier dynamics can be tuned via the lattice mismatch, which for instance plays an important role in the density of interface traps, ${ }^{18,19}$ and the valence and conduction bands offset, which determines the extent of carrier delocalization and hence the electron-hole overlap in the final core/shell NCs. ${ }^{16,17}$

To increase the radiative decay rate in semiconductor QDs, recent efforts have been directed at the growth of (quasi-)2D nanoplatelets (NPLs), typically composed of CdSe. ${ }^{20,21}$ Narrow PL spectra with full widths at half-maximum (FWHM) of 8-9 $\mathrm{nm}^{20,22}$ compared to $22-30 \mathrm{~nm}$ for CdSe QDs with similar emission wavelength (Supporting Information, SI, Figure S1), a large oscillator strength of the band-edge transition, ${ }^{20,23,24}$ and few-nanosecond PL lifetime at room temperature ${ }^{20}$ make NPLs suitable for low-threshold amplified emission and lasing, ${ }^{25-30}$ quantum emission, ${ }^{31}$ and strong light-matter interactions. ${ }^{32}$ They have been successfully assembled into long stacks ${ }^{33}$ and needles, ${ }^{34}$ leading to Förster resonance energy transfer between different NPLs that can occur on a picosecond time scale. ${ }^{35,36}$

NPL heterostructures mainly come in two forms. Core/ crown NPLs have extended lateral dimensions after shell growth while maintaining the same thickness as the original

Received: April 12, 2017

Revised: June 11, 2017

Published: June 12, 2017 
core, and exist as $\mathrm{CdSe} / \mathrm{CdS}$ type- $\mathrm{I}^{37,38}$ and $\mathrm{CdSe} / \mathrm{CdTe}$ typeII heteroNPLs. ${ }^{39}$ However, they leave a large part of their surface area exposed. Core/shell NPLs, with the shell grown on top and bottom facets, can also be synthesized ${ }^{40,41}$ and have already demonstrated high PL QEs, ${ }^{31}$ a suppressed PL intermittency (blinking), ${ }^{31}$ and applications in oxygen sensing ${ }^{42}$ or lasing. ${ }^{28-30}$ Such NPLs are, with the exception of the CdSe/ $\mathrm{Cd}_{x} \mathrm{Zn}_{1-x} \mathrm{~S}$ NPLs, ${ }^{31}$ typically based on CdSe/CdS, which, due to the small conduction band offset, are in a quasi-type-II regime with a strong electron delocalization and consequently a reduced electron-hole overlap and extended PL lifetime. ${ }^{30}$

Therefore, core/shell NPLs with a type-I band alignment would be highly desirable. Here we report on a novel heat-up procedure to synthesize a $\mathrm{ZnS}$ shell around CdSe core NPLs, yielding an improved PL QE compared to the original CdSe NPLs, and increased PL stability of NPL close-packed films under ambient conditions. We used a single-source precursor route based on zinc diethyldithiocarbamate $\left(\mathrm{Zn}(\mathrm{DDTC})_{2}\right)$, which decomposes at relatively low temperatures in the presence of amine $e^{43-45}$ so that a synthesis temperature of $100-150{ }^{\circ} \mathrm{C}$ could be maintained. The core/shell NPLs are characterized with X-ray diffractometry, transmission electron microscopy (TEM), and optical spectroscopy. Growing the shell with different precursor concentrations leads to a continuously tunable band-edge absorption and PL, with an emission that is red-shifted from 508-514 nm up to 593-611 $\mathrm{nm}$, depending on the initial core dimensions and final $\mathrm{ZnS}$ shell thickness. This trend is confirmed by $k \cdot p$ calculations, which highlight that the 2D NPL shape leads to a significant reduction of the electron and hole self-energy after $\mathrm{ZnS}$ shell growth, yielding a stronger red shift of the band edge compared to $\mathrm{CdSe} / \mathrm{ZnS}$ QDs.

\section{EXPERIMENTAL SECTION}

Materials. Zinc chloride $\left(\mathrm{ZnCl}_{2}, 99.999 \%\right)$, cadmium nitrate tetrahydrate $\left(\mathrm{Cd}\left(\mathrm{NO}_{3}\right)_{2}, 99.997 \%\right)$, cadmium(II) acetate $(99.995 \%)$, sodium diethyldithiocarbamate trihydrate (99\%), zinc diethyldithiocarbamate $\left(\mathrm{Zn}(\mathrm{DDTC})_{2}, 97 \%\right)$, carbon disulfide $\left(\mathrm{CS}_{2}, 99.9 \%\right)$, sodium sulfide $\left(\mathrm{Na}_{2} \mathrm{~S}\right)$, oleic acid $(90 \%)$, oleylamine $(70 \%)$, didodecyldimethylammonium bromide (DDAB, 98\%), formamide (FA, 99.5\%), hexane ( $\geq 95 \%)$, toluene (99.7\%), 1.2-dichlorobenzene (DCB, 99\%), acetonitrile (99.8\%), ethanol ( $\geq 99.8 \%)$, and methanol (99.9\%) were purchased from Sigma-Aldrich. N-Trioctylphosphine (TOP, 97\%) and cadmium oxide (99.999\%) were purchased from Strem Chemicals.

Cd(DDTC $)_{2}$ Preparation. Two millimolar sodium diethyldithiocarbamate was dissolved in $5 \mathrm{~mL}$ of methanol and added at room temperature to a $5 \mathrm{~mL}$ solution containing $1 \mathrm{mM} \mathrm{Cd}\left(\mathrm{NO}_{3}\right)_{2}$ in methanol while stirring. A white precipitate of cadmium diethyldithiocarbamate $\left(\mathrm{Cd}(\mathrm{DDTC})_{2}\right)$ formed, which was collected by centrifugation at $5500 \mathrm{rpm}$, rinsed three times with methanol, and dried at $60{ }^{\circ} \mathrm{C}$ for $12 \mathrm{~h}$.

ZnS Shell Growth in Toluene. First, CdSe core NPLs were synthesized according to previously reported procedures (synthesis with dry Cd-acetate). ${ }^{46}$ In a typical shell growth, for instance targeting a four monolayer (ML, $1 \mathrm{ML}$ is taken as $0.31 \mathrm{~nm}$ ) ZnS shell, a $200 \mu \mathrm{L}$, $2.26 \mu \mathrm{M},{ }^{47}$ hexane solution of CdSe NPLs (sample 1) was loaded in a $5 \mathrm{~mL}$ vial together with $2 \mathrm{~mL}$ of toluene. Next, $20 \mu \mathrm{L}$ of a mixture of $0.5 \mathrm{M} \mathrm{ZnCl}_{2}$ in oleylamine (preheated to $50{ }^{\circ} \mathrm{C}$ ), $20 \mu \mathrm{L}$ of TOP, $2 \mu \mathrm{L}$ of $\mathrm{CS}_{2}$, and $3.5 \mathrm{mg}$ of $\mathrm{Zn}(\mathrm{DDTC})_{2}$ were added. The vial was placed on a hot plate at $110^{\circ} \mathrm{C}$ (as measured with a thermocouple inserted in a reference solution), with the solution being stirred gently. After approximately $1 \mathrm{~h}$, the sample was cooled to room temperature and subsequently precipitated and redispersed three times, using $2 \mathrm{~mL}$ of acetonitrile or methanol as nonsolvent and centrifuging the sample at $5500 \mathrm{rpm}$ for $3 \mathrm{~min}$. Finally the core/shell NPLs were suspended in hexane. In case of significant conucleation of small $\mathrm{ZnS}$ clusters (SI, Figure S2), we used $n$-isopropanol to precipitate the sample and 2 min of sonication to redisperse the NPLs. After that we precipitated the NPLs again by centrifugation at $3000 \mathrm{rpm}$ for $30 \mathrm{~min}$ and finally redispersed the NPLs in hexane. To obtain different $\mathrm{ZnS}$ shell thicknesses, we varied the amounts of injected precursors. For instance, $100 \mu \mathrm{L}$ of a mixture of $0.5 \mathrm{M} \mathrm{ZnCl}_{2}$ in oleylamine (preheated to $\left.50{ }^{\circ} \mathrm{C}\right), 100 \mu \mathrm{L}$ of TOP, $10 \mu \mathrm{L}$ of $\mathrm{CS}_{2}$, and $16 \mathrm{mg}$ of $\mathrm{Zn}(\mathrm{DDTC})_{2}$ can be used to target a $12 \mathrm{ML} \mathrm{ZnS} \mathrm{shell.}$

ZnS Shell Growth in DCB. To obtain a thicker $\mathrm{ZnS}$ shell, we performed the synthesis in DCB at a temperature of $150{ }^{\circ} \mathrm{C}$. Here we describe a typical procedure, to target a $\mathrm{ZnS}$ shell of $14 \mathrm{ML}$. Other thicknesses can be obtained by injecting different amounts of precursors. CdSe NPLs dispersed in hexane were mixed with DCB at room temperature and subsequently heated under ambient atmosphere on a hot plate. When the temperature of CdSe NPLs $\left(200 \mu \mathrm{L}\right.$ at $2.26 \mu \mathrm{M}^{47}$ for sample 1) in DCB reached $150{ }^{\circ} \mathrm{C}$, we injected with a syringe pump a mixture of $120 \mu \mathrm{L}$ of $0.5 \mathrm{M} \mathrm{ZnCl}_{2}$ in oleylamine (preheated to $50^{\circ} \mathrm{C}$ ), $120 \mu \mathrm{L}$ of TOP, $12 \mu \mathrm{L}$ of $\mathrm{CS}_{2}$, and $20 \mathrm{mg}$ of $\mathrm{Zn}(\mathrm{DDTC})_{2}$ dissolved in $1 \mathrm{~mL}$ of DCB. The injection rate was set at $1 \mathrm{~mL} / \mathrm{h}$. After synthesis, we used the same purification procedure as described for the toluene-based synthesis.

CdS Shell Growth in Toluene and DCB. To grow a $6 \mathrm{ML}$ CdS shell, a $200 \mu \mathrm{L}, 2.26 \mu \mathrm{M},{ }^{47}$ hexane solution of CdSe NPLs (sample 1) was loaded in a $5 \mathrm{~mL}$ vial together with $2 \mathrm{~mL}$ of toluene. Next, $35 \mu \mathrm{L}$ of a mixture of $0.5 \mathrm{M} \mathrm{CdCl}_{2}$ in oleylamine (preheated to $100{ }^{\circ} \mathrm{C}$ ), 35 $\mu \mathrm{L}$ of TOP, $3.5 \mu \mathrm{L}$ of $\mathrm{CS}_{2}$, and $7.0 \mathrm{mg}$ of $\mathrm{Cd}(\mathrm{DDTC})_{2}$ were added. The vial was placed on a hot plate at $110{ }^{\circ} \mathrm{C}$ with the solution stirred gently. After approximately $1 \mathrm{~h}$, the sample was cooled to room temperature and subsequently precipitated as described above. Finally the NPLs were dissolved in hexane.

CdZnS Shell Growth in DCB. To obtain a CdZnS shell, we performed the synthesis in DCB at a temperature of $150{ }^{\circ} \mathrm{C}$. When the temperature of CdSe NPLs ( $200 \mu \mathrm{L}$ at $2.26 \mu \mathrm{M}$, sample 1$)$ in DCB was established, we injected, with a syringe pump, a mixture of $120 \mu \mathrm{L}$ of $0.5 \mathrm{M} \mathrm{ZnCI}_{2}$ in oleylamine (preheated up to $50{ }^{\circ} \mathrm{C}$ ), $120 \mu \mathrm{L}$ of TOP, $12 \mu \mathrm{L}$ of $\mathrm{CS}_{2}$, and $20 \mathrm{mg}$ of Cd(DDTC) $)_{2}$ dissolved in $1 \mathrm{~mL}$ of DCB. The injection rate was set at $1 \mathrm{~mL} / \mathrm{h}$. After the shell growth, the samples were purified as described above.

c-ALD Growth of ZnS and CdS Shells. CdSe/ZnS core/shell NPLs were synthesized at room temperature using colloidal atomic layer deposition (c-ALD) ${ }^{41}$ Two hundred microliters of CdSe NPLs (concentration of $2.6 \mu \mathrm{M})^{47}$ dispersed in hexane was mixed with $1 \mathrm{~mL}$ of toluene. Next $1 \mathrm{~mL}$ of a $0.1 \mathrm{M}$ solution of $\mathrm{Na}_{2} \mathrm{~S}$ in FA was added. The two-phase mixture was vigorously stirred for $15 \mathrm{~min}$ to transfer the NPLs to FA. Then the CdSe-S NPLs were precipitated with ethanol and centrifuged at $5500 \mathrm{rpm}$. After redispersion in $1 \mathrm{~mL}$ of FA, $1 \mathrm{~mL}$ of a $0.1 \mathrm{M}$ solution of DDAB in toluene was added, and the solution was stirred for $15 \mathrm{~min}$ to transfer the NPLs from FA to toluene. The NPLs were precipitated again with ethanol and redispersed in toluene. To deposit a layer of zinc, we injected $10 \mu \mathrm{L}$ of a preheated $0.5 \mathrm{M} \mathrm{ZnCl}_{2}$ solution in oleylamine and stirred for 20 min. Finally, the $\mathrm{CdSe} / \mathrm{ZnS}$ NPLs were precipitated with acetonitrile and redispersed in hexane. The procedure above was repeated until the desired number of layers was obtained, and the NPLs were finally dispersed in hexane.

$\mathrm{CdSe} / \mathrm{CdS}$ core/shell NPLs were synthesized with room temperature c-ALD. ${ }^{41,42}$ The first sulfur layer was added by transferring 300 $\mu \mathrm{L}$ of CdSe NPLs (concentration of $1 \mu \mathrm{M})^{47}$ from hexane to $1 \mathrm{~mL}$ of FA, to which $100 \mu \mathrm{L}$ of $\mathrm{Na}_{2} \mathrm{~S}(0.5 \mathrm{M})$ was added. After phase separation the polar solution was isolated. Ethanol was added to precipitate the NPLs. After centrifugation at $6000 \mathrm{rpm}$ for $1 \mathrm{~min}$, the precipitate was recovered and dried with nitrogen. Next, NPLs were redispersed in $1 \mathrm{~mL}$ of FA. The precipitation was repeated in order to remove excess sulfur. To grow the first layer of cadmium, $1 \mathrm{~mL}$ of a 0.5 $\mathrm{M}$ cadmium acetate solution in FA was added, and the solution stirred for $1 \mathrm{~h}$. The NPL dispersion was purified as described above, and the NPLs were redispersed in $1 \mathrm{~mL}$ of FA. The procedure was repeated until we reached the desirable shell thickness. The final core/shell NPLs were dispersed in $2 \mathrm{~mL}$ of toluene with the addition of $180-250$ 
$\mu \mathrm{L}$ of cadmium oleate ( $0.5 \mathrm{M}$ solution in oleic acid) to stabilize them. Excess oleic acid and cadmium oleate were removed by precipitating the NPLs with acetonitrile, centrifugation, and redispersion in toluene.

Structural Characterization. X-ray diffraction (XRD) analysis was performed on a PANanalytical Empyrean X-ray diffractometer equipped with a $1.8 \mathrm{~kW} \mathrm{CuK} \alpha$ ceramic X-ray tube, $\mathrm{PIXce}^{3 \mathrm{D}} 2 \times 2$ area detector and operating at $45 \mathrm{kV}$ and $40 \mathrm{~mA}$. Samples for the XRD measurements were prepared in a glovebox by dropping a concentrated NPL dispersion onto a miscut silicon substrate. The diffraction patterns were collected using Parallel-Beam (PB) geometry and symmetric reflection mode.

Conventional TEM images were acquired on a JEOL JEM-1011 microscope equipped with a thermionic gun at $100 \mathrm{kV}$ accelerating voltage. Samples were prepared by dropping NPL suspensions onto carbon film-coated 200 mesh copper grids. The high resolution TEM (HR-TEM) images were acquired on a $200 \mathrm{kV}$ TEM microscope (JEOL JEM-2200FS) equipped with a spherical aberration corrector (CEOS) for the objective lens, an in-column image filter ( $\Omega$-type). Samples were prepared by dropping NPLs suspensions onto holey carbon film-coated 400 mesh copper grids. High-angle annular darkfield scanning transmission electron microscope (HAADF-STEM) images were collected to measure the NPL shell thickness on 8-26 core/shell NPLs. We performed three-four measurements of the thickness along the core/shell NPLs to account for shell thickness variation. A Bruker Quantax 400 energy dispersive X-ray spectrometry (EDS) system with an XFlash 5060 detector was used for the elemental analysis. The EDS elemental maps of NPLs shown are for the $\mathrm{Cd} \mathrm{L} \alpha$, Se $\mathrm{K} \alpha, \mathrm{Zn} \mathrm{K} \alpha$, and $\mathrm{S} \mathrm{K} \alpha$ lines, respectively. Geometric phase analysis (GPA) revealed the variation in the periodicities of the HR-TEM contrast via analysis of the local components in the Fourier transform of the image. ${ }^{48}$ This method was applied to HR-TEM images of core/shell NPLs, and by selecting an "unstrained" core area in the HR-TEM image as reference, we rendered a mean dilation map.

NMR Analysis. ${ }^{1} \mathrm{H}$ nuclear magnetic resonance (NMR) spectra were recorded on a Bruker Avance III 400 NMR spectrometer, equipped with a BBI inverse probe and Z-gradients, using deuterated solvents. Toluene- $d_{8}(99.96 \%$ atom $\mathrm{D}, 1 \mathrm{~mL}$ vial) and chloroform$\mathrm{CDCl}_{3}(99.96 \%$ atom D, $1 \mathrm{~mL}$ vial) were supplied by Sigma-Aldrich. The data analysis was performed using MestReNova software version 7.1.

Optical Characterization. Absorbance spectra were recorded using a Varian Cary 3000 UV-vis spectrophotometer. Steady-state and time-resolved PL emission spectra were measured using an Edinburgh Instruments FLS920 spectrofluorometer. The steady-state PL was collected by exciting the samples at $390 \mathrm{~nm}$ with a xenon lamp. The PL decay traces were recorded by exciting the samples at $405 \mathrm{~nm}$ using a 50 ps laser diode at a repetition rate of $0.05-1 \mathrm{MHz}$, to ensure complete decay of the emission between the excitation pulses. The data were collected at the PL peak position with an emission bandwidth of $10 \mathrm{~nm}$. PL QE measurements were performed with an integrating sphere.

$\boldsymbol{k} \cdot \boldsymbol{p}$ Calculations. The NPL ground excitonic states were obtained by solving the corresponding 3D effective mass Schrödinger equation (see also SI, section theoretical outline). The Hamiltonian takes into account: (i) the anisotropy of the effective masses and their dependence on the NPL thickness; ${ }^{49}$ (ii) finite core/shell conduction and valence band offsets allowing for exciton leakage into the shell; (iii) different core and shell effective masses; and (iv) the single- and two-particle Coulomb interaction terms stemming from the dielectric mismatch between the NPLs and the organic ligands. ${ }^{50}$ The variational wave function describing the exciton reads $\Psi\left(r_{e}, r_{h}\right)=N c\left(r_{e}\right) c\left(r_{h}\right)$ $\mathrm{e}^{-\alpha\left[\left(x_{e}-x_{h}\right)^{2}+\left(y_{c}-y_{h}\right)^{2}\right]}$ with $c\left(r_{\mathrm{i}}\right)=\cos \left(k_{x} x_{i}\right) \cdot \cos \left(k_{y} y_{i}\right) \cdot \theta_{i}\left(z_{i}\right)$. In these equations, $N$ is a normalization factor, $k_{x}=\pi / L_{x}, k_{y}=\pi / L_{y}\left(L_{x}\right.$ and $L_{y}$ being the in-plane dimensions of the NPLs), and $\alpha$ is the variational parameter to optimize. The transversal $(z)$ components of the electron and hole wave functions $\theta_{i}$ were obtained numerically by means of a finite differences scheme. SI, Table S1, summarizes the values of the parameters employed in the calculations. To confirm the robustness of the results, we verified their dependence on moderate variations of the main parameters involved. By way of example, a variation of the $\mathrm{CdSe} /$ $\mathrm{ZnS}$ band offsets from $1.44 \mathrm{eV}$ (electrons) and $0.6 \mathrm{eV}$ (holes) to 1.04 and $1.0 \mathrm{eV}$ (variation of $-28 \%$ and $+67 \%$, respectively) increased (decreased) the estimated red shift (the electron-hole overlap) by no more than $6 \%$.

\section{RESULTS AND DISCUSSION}

The $\mathrm{ZnS}$ shell growth is demonstrated on CdSe NPLs that emit around $515 \mathrm{~nm}$, which are ca. $1.2 \mathrm{~nm}$ thick $^{25}$ (SI, Figure S3). As a starting point for the $\mathrm{ZnS}$ shell formation, we added 10-50 $\mu \mathrm{L}$ of a $0.5 \mathrm{M} \mathrm{ZnCl}_{2}$ solution in oleylamine and $1-5 \mu \mathrm{L}$ of $\mathrm{CS}_{2}$ to a dispersion of $200 \mu \mathrm{L}$ of $2.26 \mu \mathrm{M}$ CdSe NPLs in toluene (starting from CdSe sample 1, SI, Figure S3). When heating this mixture up to $110{ }^{\circ} \mathrm{C}$ under ambient (open air) conditions, $\mathrm{CS}_{2}$ reacts with oleylamine yielding thiocarbamic acid, which in the presence of $\mathrm{Zn}$-ions forms a $\mathrm{Zn}$-thiocarbamate salt. ${ }^{51}$ The in situ formed single-source precursor then decomposes, which can occur already at temperatures around $50-80^{\circ} \mathrm{C},{ }^{43-45}$ and forms a layer of $\mathrm{ZnS}$ around the CdSe NPLs. After shell growth, the band-edge absorption and PL spectra show a red shift of 60 nm (Figure 1 and SI, Figure S4). Note that similar, yet smaller

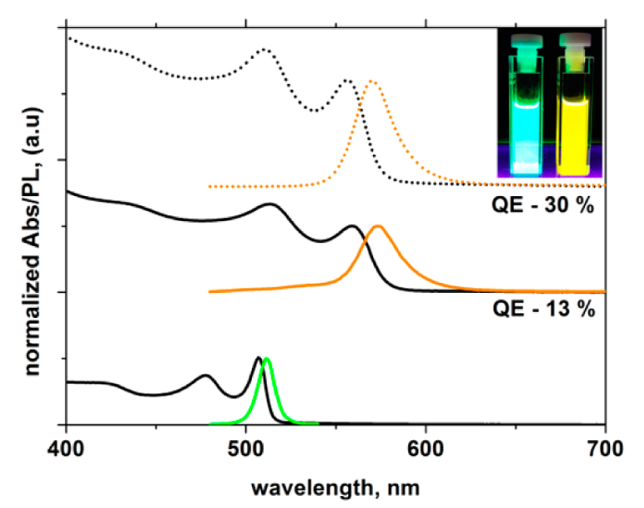

Figure 1. Absorbance and photoluminescence spectra for CdSe and $\mathrm{CdSe} / \mathrm{ZnS}$ core/shell NPLs. Bottom (full lines): Spectra of the original CdSe core NPLs (sample 1). Middle (full lines): Spectra of $\mathrm{CdSe} / \mathrm{ZnS}$ NPLs that are synthesized from a zinc oleyl-carbamic precursor produced in situ. The PL QE $\left(\lambda_{\text {ex }}=390 \mathrm{~nm}\right)$ equals $13 \%$. Top (dashed lines): Spectra of CdSe/ZnS NPLs, with a ZnS shell obtained with $\mathrm{Zn}(\mathrm{DDTC})_{2}$. The PL QE has increased to $30 \%$. Inset: Photo image of CdSe NPLs (left) and CdSe/ZnS core/shell NPLs (right, synthesized with $\left.\mathrm{Zn}(\mathrm{DDTC})_{2}\right)$, both suspended in hexane under $365 \mathrm{~nm}$ UV illumination.

red shifts have been observed previously with $\mathrm{CdSe} / \mathrm{ZnS}$ QDs, ${ }^{14} \mathrm{CdS} / \mathrm{ZnS}$ QDs, ${ }^{44}$ and CdSe NPLs passivated with Znoleate, $^{52}$ supporting the formation of the $\mathrm{ZnS}$ shell.

Instead of relying solely on the in situ generation of singlesource precursors, improved results were obtained when we replaced $50 \mathrm{~mol} \%$ of $\mathrm{CS}_{2}$ and $\mathrm{ZnCl}_{2}$ with an equivalent amount (calculated based on the $\mathrm{Zn}$-concentration) of $\mathrm{Zn}(\mathrm{DDTC})_{2}$. The inclusion of this short-chained precursor yielded a similar PL spectral position of $571 \mathrm{~nm}$ after $\mathrm{ZnS}$ shell growth, yet with sharper band-edge absorption and PL features, and a higher PL QE, which increased from $13 \%$ for the first procedure to $30 \%$ when using $\mathrm{Zn}$ (DDTC) 2 (Figure 1).

The role of the precursors was further investigated with ${ }^{1} \mathrm{H}$ NMR spectroscopy. First, as already mentioned, $\mathrm{CS}_{2}$ reacts with oleylamine to form a dithiocarbamic acid, which yields the $S$-precursor for the shell growth. Indeed, after mixing equimolar amounts of oleylamine and $\mathrm{CS}_{2}$, specific resonances at 3.7 and 
a)

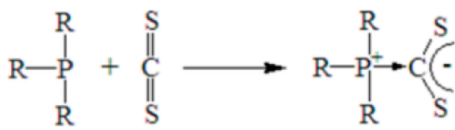

red complex

b)

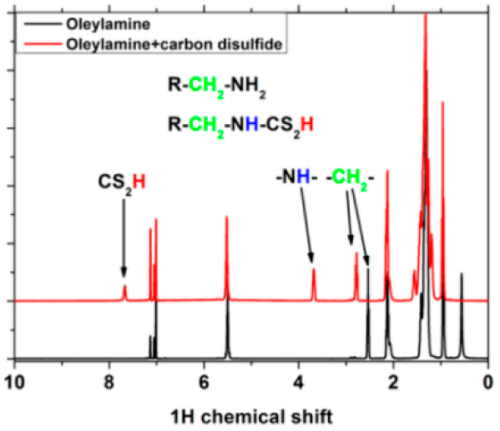

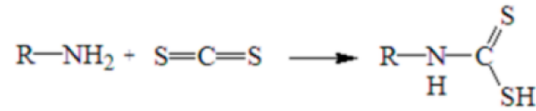

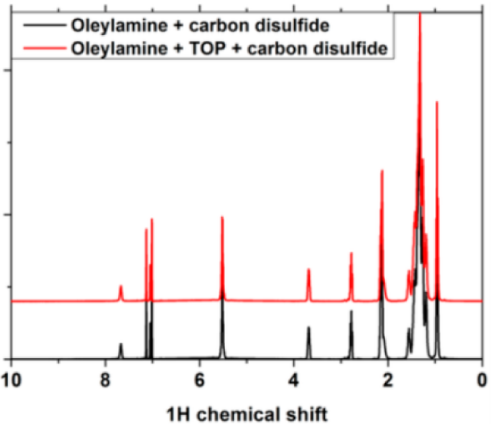

Figure 2. (a) Scheme of the chemical interactions between oleylamine, TOP, and $\mathrm{CS}_{2}$. (b) ${ }^{1} \mathrm{H}$ NMR spectra of oleylamine/CS $($ left) and oleylamine/TOP $/ \mathrm{CS}_{2}$ mixtures (right).

$7.6 \mathrm{ppm}$ appear that correspond to the $-\mathrm{NH}-$ and $-\mathrm{SH}$ protons, respectively (Figure 2). In addition, $\mathrm{CS}_{2}$ is a volatile species. However, as indicated by the saturated red color of a solution, obtained after mixing $\mathrm{CS}_{2}$ and TOP (SI, Figure S5), these two chemicals form a phosphorus-carbon ylide complex. $^{53}$ After addition of oleylamine, the solution again becomes transparent within $15 \mathrm{~min}$, and the ${ }^{1} \mathrm{H}$ NMR spectrum confirms the formation of dithiocarbamic acid (Figure 2). As the chemical shift of all NMR peaks is comparable to the first case, we can conclude that TOP serves to form an intermediate complex with $\mathrm{CS}_{2}$, which can reduce the loss of $\mathrm{CS}_{2}$ due to evaporation during synthesis, while it does not alter the formation of the final dithiocarbamic acid.

To further confirm that every precursor is required for core/ shell NPLs with high and stable PL QE, we also systematically excluded each of them during the $\mathrm{ZnS}$ shell growth. We targeted a deposition of $4 \mathrm{ML}$ of $\mathrm{ZnS}$, growing the shell via the $\mathrm{Zn}(\mathrm{DDTC})_{2}$-based heat-up reaction in toluene at $110{ }^{\circ} \mathrm{C}$. We started from CdSe NPL sample 1 , and the reaction proceeded for $1 \mathrm{~h}$ as described above. We dropcasted the resulting CdSe/ $\mathrm{ZnS}$ NPLs as a close-packed thin film onto a glass substrate, and examined their optical stability under ambient conditions for 60 days by monitoring the PL under UV illumination (Figure 3a). Absorption and PL spectra were collected on the corresponding solutions (Figure $3 \mathrm{~b}$ ). When excluding $\mathrm{ZnCl}_{2}$ or TOP, we observed a PL red shift to only 544 and $561 \mathrm{~nm}$, respectively, which indicates the growth of a thinner layer of $\mathrm{ZnS}$ around CdSe NPLs compared to the full procedure. In absence of $\mathrm{CS}_{2}$ the first excitonic peak shifted to $560 \mathrm{~nm}$; hence, the oleyl-carbamic acid precursor is not necessary to grow a thick shell. However, we did not detect any fluorescence, suggesting poor surface passivation of the latter sample possibly due to the absence of long-chained ligands. Finally, it is clear that the $\mathrm{ZnS}$ shell is most stable when it is deposited including all precursors. The red shift upon shell growth is highest in this case, reaching $567 \mathrm{~nm}$, and the closepacked thin films did not show any significant decay of the PL upon storage of the samples for 60 days.

The $\mathrm{ZnS}$ shell thickness can be varied by performing the shell growth with different concentration of $\mathrm{Zn}$ - and S-precursors (Figure 4). Targeting a given shell thickness between 2 and 14 ML, we mixed the corresponding precursors at room temperature with a $2.26 \mu \mathrm{M}$ CdSe NPL dispersion in toluene a)

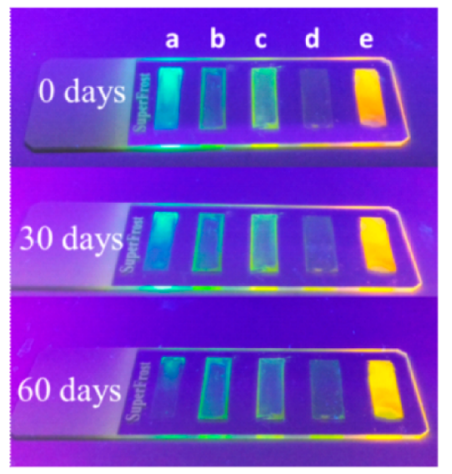

b)

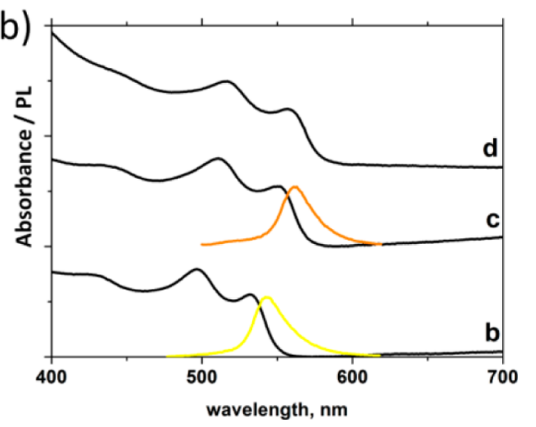

Figure 3. (a) Photo image of close-packed thin films of CdSe (sample 1 , shown as film a) NPLs with a targeted $4 \mathrm{ML} \mathrm{ZnS}$ shell, synthesized with the heat-up procedure in toluene. In each core/shell NPL synthesis, one component (b, $\mathrm{ZnCl}_{2} ; \mathrm{c}, \mathrm{TOP} ; \mathrm{d}, \mathrm{CS}_{2}$ ) was excluded. The full synthesis corresponds to the right-most film (e). The PL was monitored over time when storing the samples under ambient conditions. The $\mathrm{CdSe} / \mathrm{ZnS}$ sample that was synthesized using all precursors (film e) clearly shows the largest red shift (yellow emission), and the highest optical stability after storage for 60 days. (b) Absorbance and photoluminescence spectra of the corresponding samples (with exclusion of $\mathrm{b}, \mathrm{ZnCl}_{2} ; \mathrm{c}$, TOP; $\mathrm{d}, \mathrm{CS}_{2}$ ). In the latter case, no PL was measured.

and heated the reaction again to $110^{\circ} \mathrm{C}$. For instance, to grow a $2 \mathrm{ML}(14 \mathrm{ML}) \mathrm{ZnS}$ shell around $36.5 \mathrm{~nm}$ by $10 \mathrm{~nm} \mathrm{CdSe}$ NPLs (sample 1), we added $30 \mu \mathrm{L}(150 \mu \mathrm{L})$ of a $0.5 \mathrm{M} \mathrm{ZnCl}_{2}$ solution in oleylamine, $30 \mu \mathrm{L}(150 \mu \mathrm{L})$ of TOP, $2.5 \mu \mathrm{L}(10 \mu \mathrm{L})$ of $\mathrm{CS}_{2}$, and $2 \mathrm{mg}(100 \mathrm{mg})$ of $\mathrm{Zn}(\mathrm{DDTC})_{2}$. We used a larger relative amount of $\mathrm{Zn}(\mathrm{DDTC})_{2}$ when increasing the targeted $\mathrm{ZnS}$ shell thickness, to be able to maintain a low concentration 
a)

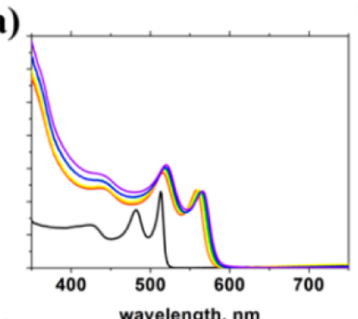

c)
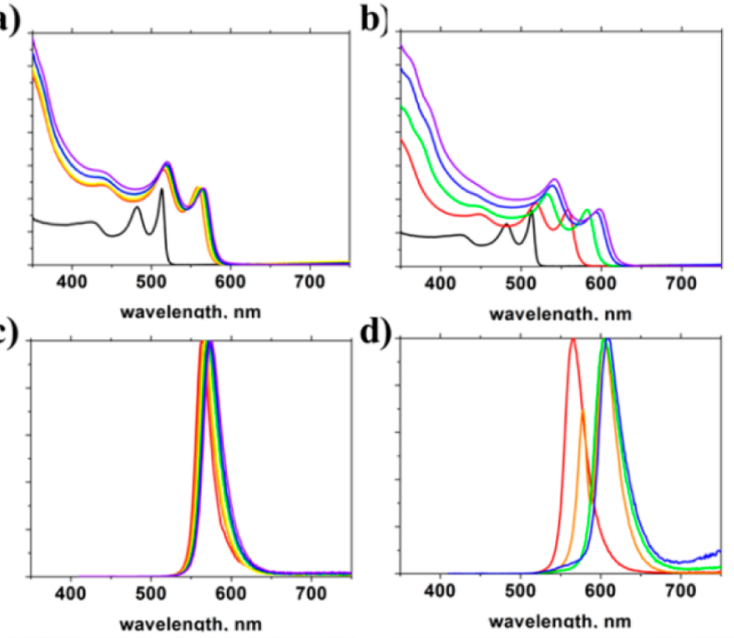

d)

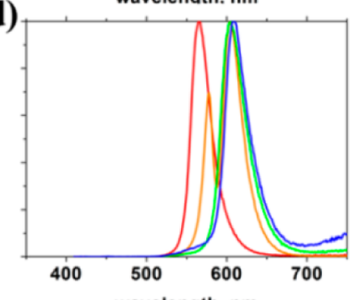

wavelenath. $\mathrm{nm}$
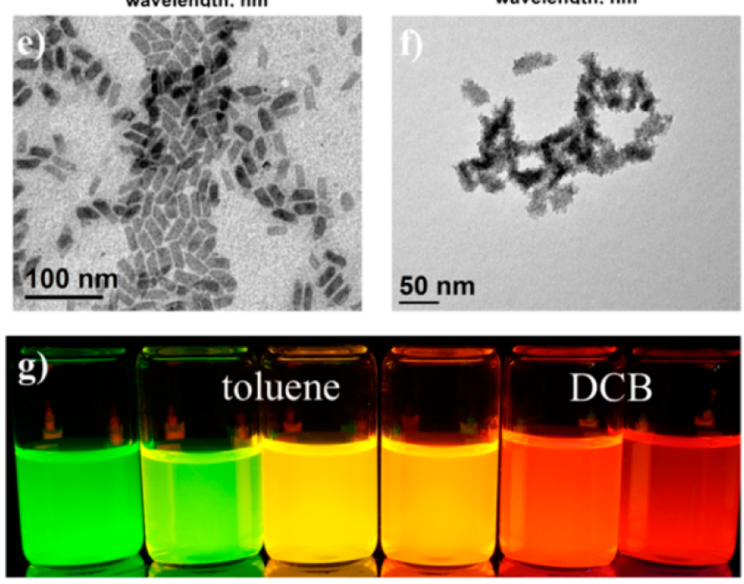

Figure 4. $(\mathrm{a}, \mathrm{b})$ Absorbance spectra for $\mathrm{CdSe}$ and $\mathrm{CdSe} / \mathrm{ZnS}$ core/ shell NPLs synthesized in toluene (a) and 1,2-dichlorobenzene (DCB) (b). (c,d) Photoluminescence spectra for CdSe/ZnS core/shell NPLs synthesized in toluene (c) and DCB (d). (e) TEM image of typical core/shell NPLs synthesized in toluene. (f) TEM image of core/shell NPLs with (targeted) $14 \mathrm{ML}$ of $\mathrm{ZnS}$ shell synthesized in DCB. (g) Image of different samples under UV illumination, from CdSe core (sample 1, green) to CdSe/ZnS core/shell NPLs with increasing ZnS shell thickness synthesized in either toluene (samples 2-4) or DCB (samples 5-6).

of volatile $\mathrm{CS}_{2}$ during shell growth when growing a thicker $\mathrm{ZnS}$ shell. The final core/shell NPLs yielded a tunable PL, with a maximal shift of $60 \mathrm{~nm}$, depending on the concentration of $\mathrm{Zn}$ and $S$ added (Figure $4 \mathrm{a}, \mathrm{c}$ ).

Still, toluene limits the growth temperature, thus possibly the $\mathrm{ZnS}$ conversion yield, as it has been demonstrated that higher growth temperatures benefit the $\mathrm{Zn}(\mathrm{DDTC})_{2}$ decomposition. ${ }^{44}$ Therefore, to further increase the shell $\mathrm{ZnS}$ thickness we performed the synthesis in DCB and raised the temperature to $150{ }^{\circ} \mathrm{C}$. To avoid separate nucleation of $\mathrm{ZnS}$, we first heated the NPLs to the final growth temperature, after which we injected the precursors continuously by syringe pump over the course of $1 \mathrm{~h}$. We used the same precursors as for the heat-up synthesis in toluene, now dissolved in DCB (see Experimental Section). With this approach, we were able to red shift the PL peak position by almost $100 \mathrm{~nm}$, to $611 \mathrm{~nm}$, when we targeted a $\mathrm{ZnS}$ shell thickness of $14 \mathrm{ML}$ (Figure $4 \mathrm{~b}, \mathrm{~d}$ ). Further analysis of the optical spectra revealed that the PL FWHM is slightly larger after shell growth, with values of $20-23 \mathrm{~nm}$ compared to $9 \mathrm{~nm}$ for CdSe core-only NPLs (Figure S3).
TEM images showed that, when using toluene as the reaction medium, the final $\mathrm{CdSe} / \mathrm{ZnS}$ NPLs preserve their original shape (Figure 4e). However, when growing a thicker $\mathrm{ZnS}$ shell in DCB, the final core/shell NPLs had a slightly more irregular shell (Figure 4f). A series of samples synthesized in DCB with different targeted shell thickness was imaged by HAADFSTEM and confirmed the irregular shell growth (SI, Figure S6), possibly induced by strain at the core/shell interface preventing epitaxial growth for thicker $\mathrm{ZnS}$ layers. From the average thickness we estimated a typical conversion yield, of precursors into $\mathrm{ZnS}$ shell, of $37 \%$, yielding an average thickness about half of the targeted value.

As a consequence of the continuous shift of the PL peak, the $\mathrm{CdSe} / \mathrm{ZnS}$ core/shell NPLs demonstrate a wide emission color range from $560 \mathrm{~nm}$ for a thin $\mathrm{ZnS}$ shell, up to $611 \mathrm{~nm}$ for the thickest $\mathrm{ZnS}$ shell (Figure 4g). To evaluate the PL QE, we also prepared two series of $\mathrm{CdSe} / \mathrm{ZnS}$ NPLs with a targeted shell thickness up to $11 \mathrm{ML}$ following the continuous injection in DCB. The PL QE increases first to values of about $60 \%$ for a targeted 3-5 ML ZnS shell, followed by a monotonic decrease when the shell thickness grows larger (SI, Figure S7, Table S2). This is likely correlated with the increase in surface roughness introduced in samples with a thicker $\mathrm{ZnS}$ shell, leading to a larger defect density.

A more detailed investigation of the $\mathrm{CdSe} / \mathrm{ZnS}$ core/shell NPLs was performed with HR-TEM, HAADF-STEM, and XRD. Zero-loss filtered HR-TEM images of core-shell NPLs with a thin shell (synthesized in toluene with a target thickness of $6 \mathrm{ML}$ ) and with a thick shell (synthesized in DCB with a target thickness of $14 \mathrm{ML}$ ) are shown in Figure 5a,c, respectively. From HAADF-STEM images of few upstanding NPLs (e.g., Figure 5e), we measured that in toluene the synthesis yields only a $2 \pm 0.5 \mathrm{ML}$ shell. In contrast, the shell obtained in DCB is much thicker (SI, Figure S6); in this particular case we even obtained $16 \pm 2 \mathrm{ML}$ of $\mathrm{ZnS}$. Here the experimental shell thickness exceeded the target value; however, considering the increased variation in shell thickness along the NPL surface, as was already apparent from the conventional TEM image (Figure 4f), we may slightly overestimate the shell thickness.

The fast Fourier transform (FFT) of core and shell regions show that the crystal phase is cubic for both CdSe and $\mathrm{ZnS}$ (Figure 5a,c), with an epitaxial relationship between core and shell, except for the outer domains of the thick-shell sample that are polycrystalline. The XRD pattern for CdSe NPLs (Figure 5f) confirms the zinc blende crystal phase, with characteristic peaks of $\mathrm{CdSe}$ at $25.2^{\circ}$ ( $\{111\}$ plane $), 41.4^{\circ}$ ( $\{220\}$ plane $)$, and $49.2^{\circ}$ ( $\{311\}$ plane $)$, at slightly smaller angles compared to bulk $\mathrm{CdSe}$, suggesting a $1.5 \%$ lattice dilation. ${ }^{54}$ After deposition of $2 \mathrm{ML}$ of $\mathrm{ZnS}$ shell, the CdSe/ $\mathrm{ZnS}$ NPLs yielded XRD peaks for the CdSe core region that are shifted to $26.7^{\circ}, 44.7^{\circ}$, and $52.7^{\circ}$, respectively, implying a $4.9 \%$ lattice contraction following shell growth due to compressive strain of the $\mathrm{ZnS}$ shell. The peaks that can be associated with the $\mathrm{ZnS}$ shell region occur at angles of $28.7^{\circ}, 47.6^{\circ}$, and $56.2^{\circ}$, respectively, which agree with bulk $\mathrm{ZnS}$. The absence of lattice dilation is possibly due to the larger bulk modulus of $\mathrm{ZnS}$ compared to CdSe. ${ }^{55}$

The large lattice mismatch between $\mathrm{CdSe}$ and $\mathrm{ZnS}$ is further highlighted by GPA (Figure 5b,d) of the HR-TEM images. By selecting an area in the core CdSe region in the HR-TEM image as a reference, the mean dilation maps clearly reveal the core-shell structure, with the CdSe region corresponding to 

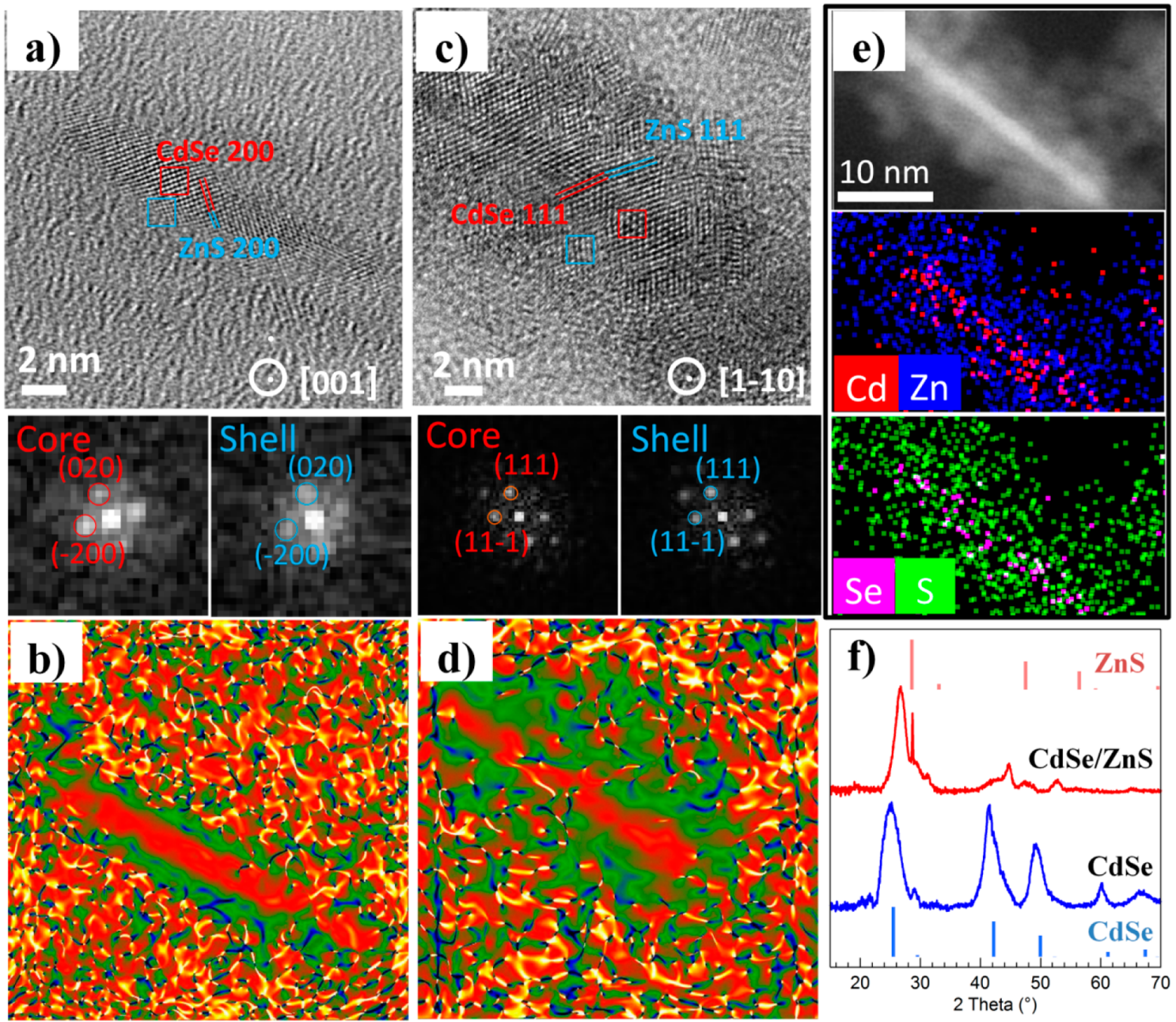

Figure 5. (a) HR-TEM image of CdSe/ZnS NPLs with a $0.6 \pm 0.2 \mathrm{~nm}(2 \pm 0.5 \mathrm{ML})$ shell, oriented with [001] zone axis, and FFT of core and shell region (boxed areas), respectively. (b) Mean dilation map obtained by the GPA method for the same image. (c) HR-TEM image of CdSe/ZnS NPLs with a $5 \pm 1 \mathrm{~nm}(16 \pm 2 \mathrm{ML})$, shell oriented with [110] zone axis, with FFT of core and shell region (boxed area), respectively. (d) Mean dilation map for the same image. (e) HAADF-STEM image and corresponding EDS map for the CdSe/ZnS sample shown in panels $c$ and d. (f) XRD patterns for CdSe core (sample 1) and corresponding CdSe/ZnS NPLs with 2 ML shell. Both core and core/shell NPLs have a cubic crystal lattice. Vertical lines indicate the positions for bulk CdSe (blue) and $\mathrm{ZnS}$ (red).

the red area and the $\mathrm{ZnS}$ shell shown in green. Note that in these measurements the relative dilation $(-12 \%$ in the green area relative to the central area) is more closely matched with the lattice mismatch between bulk CdSe and $\mathrm{ZnS}$ (12.1\%, considering $a_{\mathrm{CdSe}}=6.05 \AA$, ICSD card 620439, and $a_{\mathrm{ZnS}}=5.32$ $\AA$, ICSD card 41985). We also did not observe significant diffusion of atoms across the $\mathrm{CdSe} / \mathrm{ZnS}$ interface. Indeed, taking the $\mathrm{CdSe} / \mathrm{ZnS}$ with a $16 \mathrm{ML}$ shell, the distribution of the elements in the NPLs was mapped using EDS in Figure 5e. The EDS elemental maps confirm the GPA results of the HR-TEM images, with $\mathrm{Cd}$ and Se concentrated in the core region, while $\mathrm{Zn}$ and $\mathrm{S}$ are distributed more evenly. Note that the latter is expected for a side-view of core/shell NPLs even in absence of $\mathrm{Zn}$ and $\mathrm{S}$ diffusion into the core, as we probe a significant part of the shell when viewing the central region.

As already shown in Figure 4, by increasing the concentration of $\mathrm{Zn}$ - and S-precursors, the $\mathrm{CdSe} / \mathrm{ZnS}$ core/shell NPLs yielded a strong red shift of the PL position. To gain more insight into the nature of this shift, we monitored the evolution of the PL properties by taking aliquots during a shell growth in toluene at $110{ }^{\circ} \mathrm{C}$ (SI, Figure S8a). For this reaction, we targeted a final $\mathrm{ZnS}$ thickness of $4 \mathrm{ML}$. We observed a rapid increase of the spectral position of the heavy-hole-electron transition to $547 \mathrm{~nm}$ after $10 \mathrm{~min}$, followed by a more gradual shift in the subsequent $50 \mathrm{~min}$, reaching finally $566 \mathrm{~nm}$. For comparison, we also performed a room-temperature synthesis of CdSe/ZnS NPLs using c-ALD. The PL QE of such samples is lower in our case, with typical values of $0.5-4 \%$ (SI, Figure S9); however, the room temperature growth in sequential steps yields more control over the final shell thickness. The initial shift observed in toluene at $110{ }^{\circ} \mathrm{C}$ coincides with a $542 \mathrm{~nm}$ peak observed when $1 \mathrm{ML}$ of $\mathrm{ZnS}$ is deposited using c-ALD (SI, Figure S8b). Again, a further shift is observed when adding up to $10 \mathrm{ML}$ of $\mathrm{ZnS}$ (Figure 6, black squares, and SI, Figure S9), yielding a final red shift of $82 \mathrm{~nm}$. The shift obtained for cALD agrees well with the shift of the $\mathrm{CdSe} / \mathrm{ZnS}$ core-shell NPLs synthesized at higher temperature (Figure 6, blue triangles). As the c-ALD is executed at room temperature and the red shift in our procedure and the c-ALD are comparable, significant effects due to interface alloying can be 

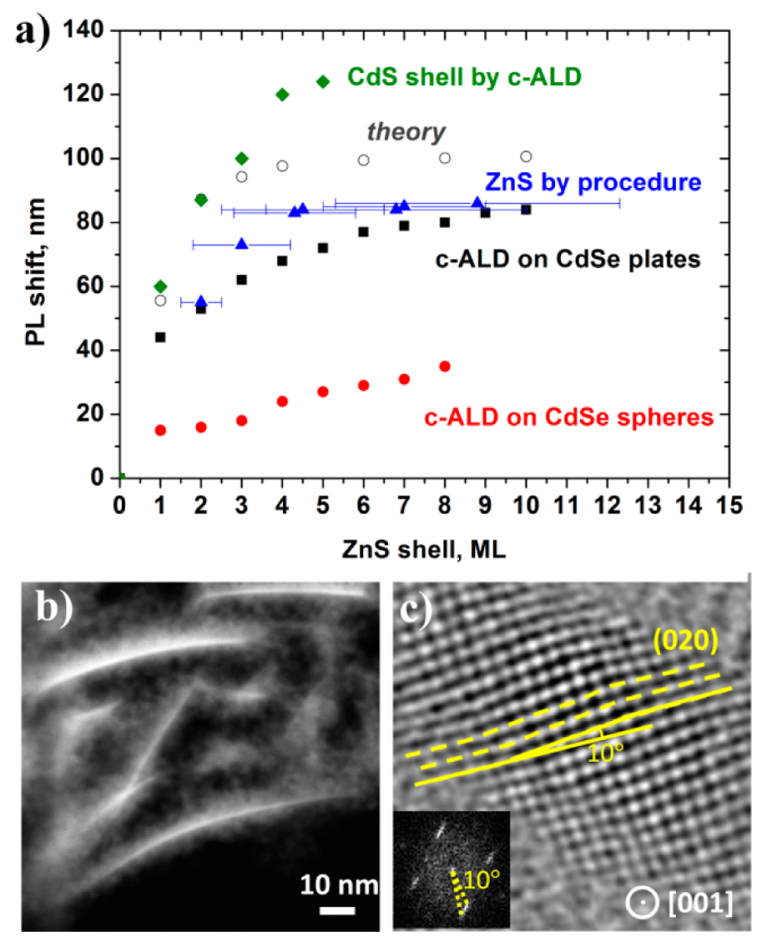

Figure 6. (a) Photoluminescence red shift for CdSe/ZnS NPLs prepared with c-ALD (black squares) and the current route at 100$150{ }^{\circ} \mathrm{C}$ (blue triangles). The horizontal error bars represent the standard deviation of the shell thickness (SI, Figure S6). For comparison, a $\mathrm{ZnS}$ shell was grown on CdSe QDs using c-ALD (red dots). Results are also compared to CdSe/CdS NPLs, synthesized with c-ALD, and $k \cdot p$ calculations of the $\mathrm{CdSe} / \mathrm{ZnS}$ exciton energy (open circles). (b) HAADF-STEM image showing a bending of CdSe/ ZnS NPLs with a $16 \mathrm{ML}$ shell. (c) Fourier-filtered HR-TEM image of $\mathrm{CdSe} / \mathrm{ZnS}$ NPLs with a $2 \mathrm{ML}$ shell, with a tilting of $10^{\circ}$ of the $\mathrm{ZnS}$ lattice relative to the $\mathrm{CdSe}$ core.

excluded in both procedures. The results are not unique to $2 \mathrm{D}$ NPLs, as CdSe/ZnS QDs, synthesized using c-ALD and with a targeted thickness of up to $8 \mathrm{ML}$, also yielded a red shift of 35 nm (Figure 6, red dots, and SI, Figure S10). The smaller value obtained for QDs, however, highlights the advantage of 2D NPLs for tuning the band gap and PL spectrum over a wide range via a type-I $\mathrm{ZnS}$ shell.

While a quantitative description is beyond the scope of the current article, we can note some factors that may contribute to the red shift of the $\mathrm{CdSe} / \mathrm{ZnS}$ band-edge absorption and emission. First, considering that the CdSe/ZnS NPLs show a strong red shift already when adding a single layer of $S$ (see also SI, Figure S8b), results suggest that, despite the type-I band alignment, the strong exciton confinement induced in $1.2 \mathrm{~nm}$ thick NPLs leads to an exciton delocalization into the $\mathrm{ZnS}$ shell and thus reduced quantum confinement along the transversal direction. Second, the PL red shift may be further induced by dielectric screening effects from the shell/ligand interface, leading to a reduction of the electron and hole self-energy. ${ }^{49}$

A calculation of the exciton confinement energy as a function of the $\mathrm{ZnS}$ shell growth confirmed these trends. Within the framework of single-band $k \cdot p$ theory, we numerically calculated the electron and hole confinement energies and wave functions in the transversal direction, taking into account the finite $\mathrm{CdSe} /$ $\mathrm{ZnS}$ band offset and the Coulomb self-polarization contribution that stems from the NPL/ligand dielectric contrast. ${ }^{50}$ Then, following a variational procedure (see SI, theoretical section), we obtained the in-plane contributions to the exciton energy and wave function. These included the polarization of the Coulomb interaction owing to the dielectric mismatch ${ }^{50}$ and the effects of the reduced dimensionality of the CdSe NPLs on the electron and hole effective masses. ${ }^{49}$ The calculations yielded an electron and hole wave function tunneling into the $\mathrm{ZnS}$ shell (SI, Figure S11e) and a consequent red shift of the emission wavelength with increasing shell thickness, in agreement with the experimental findings (Figure 6a, open circles). The larger magnitude of the red shift as compared to the CdSe/ZnS QDs (Figure 6, dots) can be mainly ascribed to the $1.2 \mathrm{~nm}$ NPL thickness. Despite the large CdSe/ZnS band offset, the strong confinement in this dimension increases significantly the single particle band gap, promoting tunneling of the electron and hole into the shell. A more thorough analysis revealed, however, that the delocalization of the exciton into the $\mathrm{ZnS}$ shell is not sufficient to account for the strong PL red shift (SI, Figure S11a). We found that the discontinuity of the dielectric screening at the NPLs surface further influences the electron and hole wave functions and associated exciton energy (SI, Figure S11d), giving rise to (i) a notable increase of the calculated red shifts for each $\mathrm{ZnS}$ shell thickness, and (ii) an enhanced electron-hole correlation. Both effects stem from the extreme anisotropy of the NPL geometry. In the first case, such anisotropy hinders the mutual compensation of surface polarization and self-polarization terms, extensively reported for excitons in QDs. ${ }^{56-59}$ This distinctive feature of NPLs explains their stronger PL red shift after shell growth as compared to QDs. Indeed, in NPLs, the attraction of the electron and hole image charges compensates only partially the dominating repulsive effect of single-particle self-energies. ${ }^{60}$ The difference between these contributions decreases when the extreme anisotropy of the dielectric confinement is relieved by growing the $\mathrm{ZnS}$ shell and moving the NPL-ligands dielectric interface further out. The overall effect is a reduction of the (net) repulsive contribution of the dielectric effects as the $\mathrm{ZnS}$ shell grows thicker, and a concomitant PL red shift. In the second case, the weak in-plane confinement turns the exciton wave function very reactive against changes in the electronhole interaction potential, which is highly influenced by the strong dielectric confinement in the transversal direction. The immediate consequences are large electron-hole overlaps (SI, Figure $S 11 b-d$ ) and thus short PL lifetimes. In this regard, the calculations predict a decrease of the overlap, up to $50 \%$, as a function of the shell thickness (SI, Figure S11b), which is a direct consequence of relaxing the transversal dielectric confinement. The calculations were corroborated by a measurement of the PL decay trace of samples with varying shell thickness (SI, Figure S7 and Table S2). In line with the reduced electron-hole overlap, after $\mathrm{ZnS}$ shell growth we observed an extension of the average PL decay time.

Finally, as already suggested by the XRD and HR-TEM data, due to the $1.2 \mathrm{~nm}$ CdSe NPL thickness and the large lattice mismatch between $\mathrm{CdSe}$ and $\mathrm{ZnS}$, strain may also build up inside the CdSe core. The presence of strain in our structures is further apparent from a HAADF-STEM image for $\mathrm{CdSe} / \mathrm{ZnS}$ core/shell NPLs (Figure 6b), where we observed a bending of the CdSe/ZnS NPLs. Moreover, Fourier-filtered HR-TEM images (Figure $6 \mathrm{c}, \mathrm{CdSe} / \mathrm{ZnS}$ with a $2 \mathrm{ML} \mathrm{ZnS}$ shell) demonstrate that the CdSe and $\mathrm{ZnS}[100]$ crystal directions are slightly misaligned. These strain-induced CdSe lattice modifications and overall bending of the NPLs may cause a further correction to the PL red shift, the latter similar to the reduction 
of the band edge already observed in elastically bent $\mathrm{CdS}$ nanowires. $^{61}$

Next to the results presented above, the shell growth procedure can also be expanded to $\mathrm{CdS}$ and $\mathrm{CdZnS}$ shells. For instance, a CdS shell can be grown in toluene at $100-110{ }^{\circ} \mathrm{C}$, using only $\mathrm{CdCl}_{2}$ and equimolar amount of $\mathrm{CS}_{2}$ in the presence of TOP (SI, Figure S12). As the valence band offset is strongly reduced in $\mathrm{CdSe} / \mathrm{CdS}$ core/shell NPLs, these samples displayed a significant red shift of the PL maximum, up to $620 \mathrm{~nm}$ when targeting a shell thickness of $6 \mathrm{ML}$ (in line with the large red shift observed when applying a CdS shell by cALD, Figure 6, green diamonds). However, when growing the shell with this procedure, CdS also nucleated on the edges of the CdSe NPLs as small clusters (SI, Figure S12c). When applying a continuous injection synthesis in $\mathrm{DCB}$ at $150{ }^{\circ} \mathrm{C}$ to deposit a CdS shell using also $\mathrm{Cd}(\mathrm{DDTC})_{2}$, these clusters progressed into thin $\mathrm{CdS}$ nanorods around the CdSe NPL edges (SI, Figure S12d), similar to previously published results on $2 \mathrm{D}$ nanocrystals. ${ }^{62}$

The best results were achieved by exchanging $\mathrm{CdCl}_{2}$ for $\mathrm{ZnCl}_{2}$ while keeping $\mathrm{Cd}(\mathrm{DDTC})_{2}$ as the $\mathrm{CdS}$ single-source precursor (at a $\mathrm{Cd}: \mathrm{Zn}$ ratio of 1:1). Applying again the continuous injection in $\mathrm{DCB}$, we observed a stronger red shift of the PL compared to CdSe/ZnS NPLs (Figure 7), despite the

a)

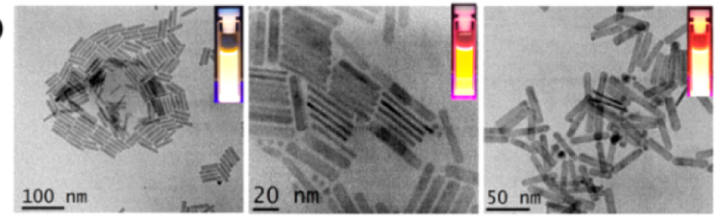

b)

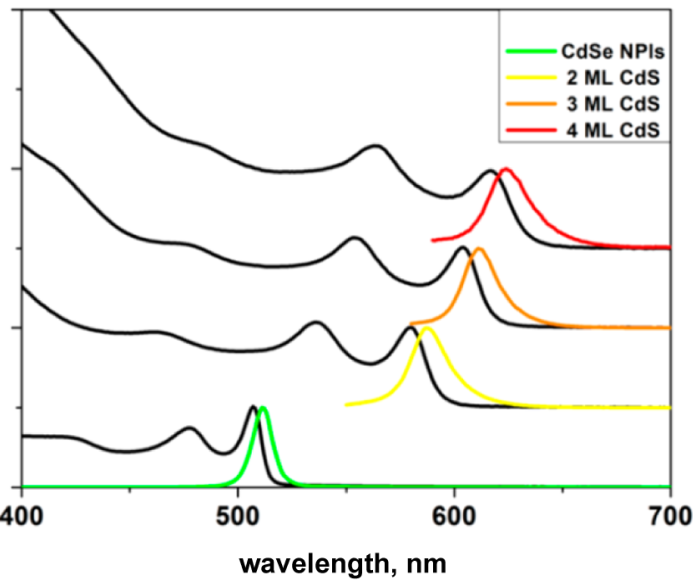

Figure 7. (a) TEM images of $\mathrm{CdSe} / \mathrm{CdZnS}$ core-shell NPLs with a targeted thickness of 2,3 , and $4 \mathrm{ML}$, using $\mathrm{ZnCl}_{2}$ and $\mathrm{Cd}(\mathrm{DDTC})_{2}$. The inset shows the NPL dispersion under UV illumination. (b) Corresponding absorbance and photoluminescence spectra.

inclusion of $\mathrm{ZnCl}_{2}$ in the synthesis. The PL QE of these samples was raised to $58 \%$. More importantly, the shell was deposited more homogeneously over the $\mathrm{CdSe}$ core (Figure $7 \mathrm{a}$ ), possibly due to the reduced reactivity of $\mathrm{ZnCl}_{2}$ compared to $\mathrm{CdCl}_{2}$, yielding a more controlled growth.

\section{CONCLUSIONS}

We demonstrated a robust procedure for coating CdSe NPLs with $\mathrm{ZnS}, \mathrm{CdS}$, and $\mathrm{CdZnS}$, using toluene or $\mathrm{DCB}$ as a solvent. The procedure relies on a room temperature injection of precursors followed by heat up or by slow precursor injection at elevated temperatures, and the entire shell growth was performed under open air conditions, demonstrating that synthesis under $\mathrm{N}_{2}$ atmosphere is not required to obtain highquality core/shell NPLs. Consequently, it should lend itself well to scale-up. HAAFD-STEM images yielded CdSe/ZnS NPLs with a shell thickness up to $9 \mathrm{ML}$. Our method yielded CdSe/ $\mathrm{ZnS}$ and $\mathrm{CdSe} / \mathrm{CdZnS}$ NPLs with a PL QE up to $64 \%$ and $58 \%$, respectively. Despite the type-I band alignment in CdSe/ $\mathrm{ZnS}$ core-shell NPLs, we observed a significant red shift after shell growth, which could be attributed to exciton delocalization into the $\mathrm{ZnS}$ due to strong vertical confinement in $1.2 \mathrm{~nm}$ thick CdSe NPLs, as well as a modification of the Coulomb interactions that reduces the electron and hole self-energy. The final samples show excellent stability under ambient conditions, which opens up the way toward practical photonic and optoelectronic applications of CdSe-based 2D NPLs.

\section{ASSOCIATED CONTENT}

\section{S Supporting Information}

The Supporting Information is available free of charge on the ACS Publications website at DOI: 10.1021/acs.chemmater.7b01513.

Absorption and PL spectra of CdSe core and CdSe/ZnS core/shell QDs. Optical spectra and TEM image of CdSe core NPLs. Additional optical spectra of $\mathrm{CdSe} / \mathrm{ZnS}$ NPLs synthesized by present procedure and room temperature c-ALD. Absorbance spectra of precursors. PL QEs of different CdSe/ZnS NPL samples. Additional details of the $k \cdot p$ calculations and plots of the exciton wave function. Additional optical spectra and TEM images for the CdSe/CdS NPLs (PDF)

\section{AUTHOR INFORMATION}

\section{Corresponding Author}

*E-mail: iwan.moreels@iit.it.

ORCID

Ali Hossain Khan: 0000-0001-7155-0200

Iwan Moreels: 0000-0003-3998-7618

Present Address

"CEA Saclay, 91191 Gif-sur-Yvette, France.

\section{Author Contributions}

The manuscript was written through contributions of all authors. All authors have given approval to the final version of the manuscript.

\section{Funding}

The present publication is realized with the support of the Ministero degli Affari Esteri e della Cooperazione Internazionale (IONX-NC4SOL). This project has also received funding from the European Union's Horizon 2020 research and innovation program under Grant Agreement No. 696656 (GrapheneCore1). J.L.M. acknowledges support from UJI project P1-1B2014-24 and MINECO project CTQ201460178-P.

\section{Notes}

The authors declare no competing financial interest.

\section{ACKNOWLEDGMENTS}

We thank J. I. Climente and J. Planelles (University Jaume I, Spain) for fruitful discussions. 


\section{REFERENCES}

(1) Yoffe, A. D. Low-Dimensional Systems: Quantum Size Effects and Electronic Properties of Semiconductor Microcrystallites (ZeroDimensional Systems) and Some Quasi-Two-Dimensional Systems. Adv. Phys. 1993, 42, 173-262.

(2) Yin, Y.; Alivisatos, A. P. Colloidal Nanocrystal Synthesis and the Organic-Inorganic Interface. Nature 2005, 437, 664-670.

(3) Kovalenko, M. V.; Manna, L.; Cabot, A.; Hens, Z.; Talapin, D. V.; Kagan, C. R.; Klimov, V. I.; Rogach, A. L.; Reiss, P.; Milliron, D. J.; Guyot-Sionnnest, P.; Konstantatos, G.; Parak, W. J.; Hyeon, T.; Korgel, B. A.; Murray, C. B.; Heiss, W. Prospects of Nanoscience with Nanocrystals. ACS Nano 2015, 9, 1012-1057.

(4) Lo, S. S.; Mirkovic, T.; Chuang, C.-H.; Burda, C.; Scholes, G. D. Emergent Properties Resulting from Type-II Band Alignment in Semiconductor Nanoheterostructures. Adv. Mater. 2011, 23, 180-197.

(5) Fernée, M. J.; Tamarat, P.; Lounis, B. Spectroscopy of Single Nanocrystals. Chem. Soc. Rev. 2014, 43, 1311-1337.

(6) Kambhampati, P. Multiexcitons in Semiconductor Nanocrystals: A Platform for Optoelectronics at High Carrier Concentration. J. Phys. Chem. Lett. 2012, 3, 1182-1190.

(7) Pietryga, J. M.; Park, Y.-S.; Lim, J.; Fidler, A. F.; Bae, W. K.; Brovelli, S.; Klimov, V. I. Spectroscopic and Device Aspects of Nanocrystal Quantum Dots. Chem. Rev. 2016, 116, 10513-10622.

(8) Kagan, C. R.; Lifshitz, E.; Sargent, E. H.; Talapin, D. V. Building Devices from Colloidal Quantum Dots. Science 2016, 353, aac5523.

(9) Grim, J. Q.; Manna, L.; Moreels, I. A Sustainable Future for Photonic Colloidal Nanocrystals. Chem. Soc. Rev. 2015, 44, 58975914.

(10) Dai, X.; Zhang, Z.; Jin, Y.; Niu, Y.; Cao, H.; Liang, X.; Chen, L.; Wang, J.; Peng, X. Solution-Processed, High-Performance LightEmitting Diodes Based on Quantum Dots. Nature 2014, 515, 96-99.

(11) Carey, G. H.; Abdelhady, A. L.; Ning, Z.; Thon, S. M.; Bakr, O. M.; Sargent, E. H. Colloidal Quantum Dot Solar Cells. Chem. Rev. 2015, 115, 12732-12763.

(12) Saran, R.; Curry, R. J. Lead Sulphide Nanocrystal Photodetector Technologies. Nat. Photonics 2016, 10, 81-92.

(13) Zhou, J.; Yang, Y.; Zhang, C. Toward Biocompatible Semiconductor Quantum Dots: From Biosynthesis and Bioconjugation to Biomedical Application. Chem. Rev. 2015, 115, 11669-11717.

(14) Hines, M. A.; Guyot-Sionnest, P. Synthesis and Characterization of Strongly Luminescing ZnS-Capped CdSe Nanocrystals. J. Phys. Chem. 1996, 100, 468-471.

(15) Talapin, D. V.; Mekis, I.; Götzinger, S.; Kornowski, A.; Benson, O.; Weller, H. CdSe/CdS/ZnS and CdSe/ZnSe/ZnS Core-ShellShell Nanocrystals. J. Phys. Chem. B 2004, 108, 18826-18831.

(16) Mahler, B.; Spinicelli, P.; Buil, S.; Quelin, X.; Hermier, J.-P.; Dubertret, B. Towards Non-Blinking Colloidal Quantum Dots. Nat. Mater. 2008, 7, 659-664.

(17) Chen, Y.; Vela, J.; Htoon, H.; Casson, J. L.; Werder, D. J.; Bussian, D. A.; Klimov, V. I.; Hollingsworth, J. A. Giant” Multishell CdSe Nanocrystal Quantum Dots with Suppressed Blinking. J. Am. Chem. Soc. 2008, 130, 5026-5027.

(18) Bae, W. K.; Padilha, L. A.; Park, Y.-S.; McDaniel, H.; Robel, I.; Pietryga, J. M.; Klimov, V. I. Controlled Alloying of the Core-Shell Interface in CdSe/CdS Quantum Dots for Suppression of Auger Recombination. ACS Nano 2013, 7, 3411-3419.

(19) Christodoulou, S.; Vaccaro, G.; Pinchetti, V.; de Donato, F.; Grim, J. Q.; Casu, A.; Genovese, A.; Vicidomini, G.; Diaspro, A.; Brovelli, S.; Manna, L.; Moreels, I. Synthesis of Highly Luminescent Wurtzite CdSe/CdS Giant-Shell Nanocrystals Using a Fast Continuous Injection Route. J. Mater. Chem. C 2014, 2, 3439-3447.

(20) Ithurria, S.; Tessier, M. D.; Mahler, B.; Lobo, R. P. S. M.; Dubertret, B.; Efros, A. L. Colloidal Nanoplatelets with TwoDimensional Electronic Structure. Nat. Mater. 2011, 10, 936-941.

(21) Lhuillier, E.; Pedetti, S.; Ithurria, S.; Nadal, B.; Heuclin, H.; Dubertret, B. Two-Dimensional Colloidal Metal Chalcogenides Semiconductors: Synthesis, Spectroscopy, and Applications. Acc. Chem. Res. 2015, 48, 22-30.
(22) Zhang, F.; Wang, S.; Wang, L.; Lin, Q.; Shen, H.; Cao, W.; Yang, C.; Wang, H.; Yu, L.; Du, Z.; Xue, J.; Li, L. S. Super Color Purity Green Quantum Dot Light-Emitting Diodes Fabricated by Using CdSe/CdS Nanoplatelets. Nanoscale 2016, 8, 12182-12188.

(23) Naeem, A.; Masia, F.; Christodoulou, S.; Moreels, I.; Borri, P.; Langbein, W. Giant Exciton Oscillator Strength and Radiatively Limited Dephasing in Two-Dimensional Platelets. Phys. Rev. B: Condens. Matter Mater. Phys. 2015, 91, 121302.

(24) Achtstein, A. W.; Schliwa, A.; Prudnikau, A.; Hardzei, M.; Artemyev, M. V.; Thomsen, C.; Woggon, U. Electronic Structure and Exciton-Phonon Interaction in Two-Dimensional Colloidal CdSe Nanosheets. Nano Lett. 2012, 12, 3151-3157.

(25) She, C.; Fedin, I.; Dolzhnikov, D. S.; Demortière, A.; Schaller, R. D.; Pelton, M.; Talapin, D. V. Low-Threshold Stimulated Emission Using Colloidal Quantum Wells. Nano Lett. 2014, 14, 2772-2777.

(26) Grim, J. Q.; Christodoulou, S.; Di Stasio, F.; Krahne, R.; Cingolani, R.; Manna, L.; Moreels, I. Continuous-Wave Biexciton Lasing at Room Temperature Using Solution-Processed Quantum Wells. Nat. Nanotechnol. 2014, 9, 891-895.

(27) Guzelturk, B.; Kelestemur, Y.; Olutas, M.; Delikanli, S.; Demir, H. V. Amplified Spontaneous Emission and Lasing in Colloidal Nanoplatelets. ACS Nano 2014, 8, 6599-6605.

(28) Li, M.; Zhi, M.; Zhu, H.; Wu, W.-Y.; Xu, Q.-H.; Jhon, M. H.; Chan, Y. Ultralow-Threshold Multiphoton-Pumped Lasing from Colloidal Nanoplatelets in Solution. Nat. Commun. 2015, 6, 8513.

(29) She, C.; Fedin, I.; Dolzhnikov, D. S.; Dahlberg, P. D.; Engel, G. S.; Schaller, R. D.; Talapin, D. V. Red, Yellow, Green, and Blue Amplified Spontaneous Emission and Lasing Using Colloidal CdSe Nanoplatelets. ACS Nano 2015, 9, 9475-9485.

(30) Kelestemur, Y.; Guzelturk, B.; Erdem, O.; Olutas, M.; Gungor, K.; Demir, H. V. Platelet-in-Box Colloidal Quantum Wells: CdSe/ CdS@CdS Core/Crown@Shell Heteronanoplatelets. Adv. Funct. Mater. 2016, 26, 3570-3579.

(31) Tessier, M. D.; Mahler, B.; Nadal, B.; Heuclin, H.; Pedetti, S.; Dubertret, B. Spectroscopy of Colloidal Semiconductor Core/Shell Nanoplatelets with High Quantum Yield. Nano Lett. 2013, 13, 33213328.

(32) Flatten, L. C.; Christodoulou, S.; Patel, R. K.; Buccheri, A.; Coles, D. M.; Reid, B. P. L.; Taylor, R. A.; Moreels, I.; Smith, J. M. Strong Exciton-Photon Coupling with Colloidal Nanoplatelets in an Open Microcavity. Nano Lett. 2016, 16, 7137-7141.

(33) Jana, S.; Davidson, P.; Abécassis, B. CdSe Nanoplatelets: Living Polymers. Angew. Chem., Int. Ed. 2016, 55, 9371-9374.

(34) Abécassis, B.; Tessier, M. D.; Davidson, P.; Dubertret, B. SelfAssembly of CdSe Nanoplatelets into Giant Micrometer-Scale Needles Emitting Polarized Light. Nano Lett. 2014, 14, 710-715.

(35) Rowland, C. E.; Fedin, I.; Zhang, H.; Gray, S. K.; Govorov, A. O.; Talapin, D. V.; Schaller, R. D. Picosecond Energy Transfer and Multiexciton Transfer Outpaces Auger Recombination in Binary CdSe Nanoplatelet Solids. Nat. Mater. 2015, 14, 484-489.

(36) Moreels, I. Colloidal Nanoplatelets: Energy Transfer Is Speeded up in 2D. Nat. Mater. 2015, 14, 464-465.

(37) Prudnikau, A.; Chuvilin, A.; Artemyev, M. CdSe-CdS Nanoheteroplatelets with Efficient Photoexcitation of Central CdSe Region through Epitaxially Grown CdS Wings. J. Am. Chem. Soc. 2013, $135,14476-14479$

(38) Li, Q.; Wu, K.; Chen, J.; Chen, Z.; McBride, J. R; Lian, T. SizeIndependent Exciton Localization Efficiency in Colloidal CdSe/CdS Core/Crown Nanosheet Type-I Heterostructures. ACS Nano 2016, 10, 3843-3851.

(39) Pedetti, S.; Ithurria, S.; Heuclin, H.; Patriarche, G.; Dubertret, B. Type-II CdSe/CdTe Core/Crown Semiconductor Nanoplatelets. J. Am. Chem. Soc. 2014, 136, 16430-16438.

(40) Mahler, B.; Nadal, B.; Bouet, C.; Patriarche, G.; Dubertret, B. Core/shell Colloidal Semiconductor Nanoplatelets. J. Am. Chem. Soc. 2012, 134, 18591-18598.

(41) Ithurria, S.; Talapin, D. V. Colloidal Atomic Layer Deposition (c-ALD) Using Self-Limiting Reactions at Nanocrystal Surface 
Coupled to Phase Transfer between Polar and Nonpolar Media. J. Am. Chem. Soc. 2012, 134, 18585-18590.

(42) Lorenzon, M.; Christodoulou, S.; Vaccaro, G.; Pedrini, J.; Meinardi, F.; Moreels, I.; Brovelli, S. Reversed Oxygen Sensing Using Colloidal Quantum Wells towards Highly Emissive Photoresponsive Varnishes. Nat. Commun. 2015, 6, 6434.

(43) Pradhan, N.; Katz, B.; Efrima, S. Synthesis of High-Quality Metal Sulfide Nanoparticles from Alkyl Xanthate Single Precursors in Alkylamine Solvents. J. Phys. Chem. B 2003, 107, 13843-13854.

(44) Chen, D.; Zhao, F.; Qi, H.; Rutherford, M.; Peng, X. Bright and Stable Purple/Blue Emitting CdS/ZnS Core/Shell Nanocrystals Grown by Thermal Cycling Using a Single-Source Precursor. Chem. Mater. 2010, 22, 1437-1444.

(45) Jung, Y. K.; Kim, J. I.; Lee, J.-K. Thermal Decomposition Mechanism of Single-Molecule Precursors Forming Metal Sulfide Nanoparticles. J. Am. Chem. Soc. 2010, 132, 178-184.

(46) Bertrand, G. H. V.; Polovitsyn, A.; Christodoulou, S.; Khan, A. H.; Moreels, I. Shape Control of Zincblende CdSe Nanoplatelets. Chem. Commun. 2016, 52, 11975-11978.

(47) Achtstein, A. W.; Antanovich, A.; Prudnikau, A.; Scott, R.; Woggon, U.; Artemyev, M. Linear Absorption in CdSe Nanoplates: Thickness and Lateral Size Dependency of the Intrinsic Absorption. J. Phys. Chem. C 2015, 119, 20156-20161.

(48) Hÿtch, M. J.; Snoeck, E.; Kilaas, R. Quantitative Measurement of Displacement and Strain Fields from HREM Micrographs. Ultramicroscopy 1998, 74, 131-146.

(49) Benchamekh, R.; Gippius, N. A.; Even, J.; Nestoklon, M. O.; Jancu, J.-M.; Ithurria, S.; Dubertret, B.; Efros, A. L.; Voisin, P. TightBinding Calculations of Image-Charge Effects in Colloidal Nanoscale Platelets of CdSe. Phys. Rev. B: Condens. Matter Mater. Phys. 2014, 89, 35307.

(50) Kumagai, M.; Takagahara, T. Excitonic and Nonlinear-Optical Properties of Dielectric Quantum-Well Structures. Phys. Rev. B: Condens. Matter Mater. Phys. 1989, 40, 12359-12381.

(51) Purdy, A. P.; George, C. F. Anhydrous Dithiocarbamates. Potential Precursors to Alkaline Earth Sulfide Materials. Main Group Chem. 1996, 1, 229-240.

(52) Zhou, Y.; Wang, F.; Buhro, W. E. Large Exciton Energy Shifts by Reversible Surface Exchange in 2D II-VI Nanocrystals. J. Am. Chem. Soc. 2015, 137, 15198-15208.

(53) Bianchini, C.; Meli, A.; Orlandini, A. Reactivity of the Triethylphosphine-Carbon Disulfide Adduct (Et3P.CS2) toward cobalt(II) Cations in the Presence of the Tris (Tertiary Phosphines) Triphos and Etriphos. X-Ray Crystal Structure of the Complex [(etriphos)Co(S2C(H)PEt3)](BPh4)2. Inorg. Chem. 1982, 21, 41614165 .

(54) Chen, D.; Gao, Y.; Chen, Y.; Ren, Y.; Peng, X. Structure Identification of Two-Dimensional Colloidal Semiconductor Nanocrystals with Atomic Flat Basal Planes. Nano Lett. 2015, 15, 44774482.

(55) Madelung, O., Rössler, U., Schulz, M., Eds. II-VI and I-VII Compounds; Semimagnetic Compounds; Landolt-Börnstein: Group III Condensed Matter; Springer-Verlag: Berlin/Heidelberg, 1999; Vol. $41 B$.

(56) Brus, L. E. Electron-electron and Electron-hole Interactions in Small Semiconductor Crystallites: The Size Dependence of the Lowest Excited Electronic State. J. Chem. Phys. 1984, 80, 4403-4409.

(57) Lannoo, M.; Delerue, C.; Allan, G. Screening in Semiconductor Nanocrystallites and Its Consequences for Porous Silicon. Phys. Rev. Lett. 1995, 74, 3415-3418.

(58) Bolcatto, P. G.; Proetto, C. R. Shape and Dielectric Mismatch Effects in Semiconductor Quantum Dots. Phys. Rev. B: Condens. Matter Mater. Phys. 1999, 59, 12487-12498.

(59) Fonoberov, V. A.; Pokatilov, E. P.; Balandin, A. A. Exciton States and Optical Transitions in Colloidal CdS Quantum Dots: Shape and Dielectric Mismatch Effects. Phys. Rev. B: Condens. Matter Mater. Phys. 2002, 66, 85310.
(60) Rodina, A. V.; Efros, A. L. Effect of Dielectric Confinement on Optical Properties of Colloidal Nanostructures. J. Exp. Theor. Phys. 2016, 122, 554-566.

(61) Zhang, C.; Cretu, O.; Kvashnin, D. G.; Kawamoto, N.; Mitome, M.; Wang, X.; Bando, Y.; Sorokin, P. B.; Golberg, D. Statistically Analyzed Photoresponse of Elastically Bent CdS Nanowires Probed by Light-Compatible In Situ High-Resolution TEM. Nano Lett. 2016, 16, 6008-6013.

(62) Wu, X.-J.; Chen, J.; Tan, C.; Zhu, Y.; Han, Y.; Zhang, H. Controlled Growth of High-Density CdS and CdSe Nanorod Arrays on Selective Facets of Two-Dimensional Semiconductor Nanoplates. Nat. Chem. 2016, 8, 470-475. 